\title{
Difficulties when using video playback to investigate social cognition in California scrub-jays (Aphelocoma californica)
}

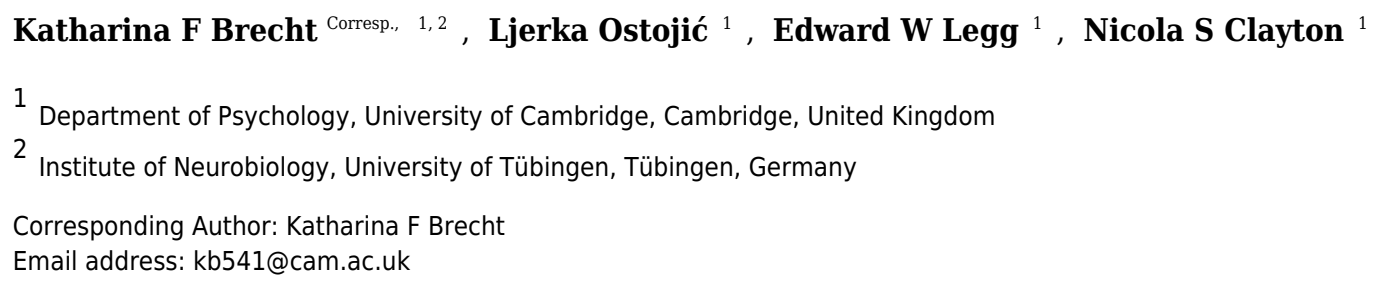

Previous research has suggested that videos can be used to experimentally manipulate social stimuli. In the present study, we used the California scrub-jays' cache protection strategies to assess whether video playback can be used to simulate conspecifics in a social context. In both the lab and the field, scrub-jays are known to exhibit a range of behaviours to protect their caches from potential pilferage by a conspecific, for example by hiding food in locations out of the observer's view or by re-caching previously made caches once the observer has left. Here, we presented scrub-jays with videos of a conspecific observer as well as two non-social conditions during a caching period and assessed whether they would cache out of the observer's "view" (Experiment 1) or would re-cache their caches once the observer was no longer present (Experiment 2). In contrast to previous studies using live observers, the scrub-jays' caching and re-caching behaviour was not influenced by whether the observer was present or absent. These findings suggest that there might be limitations in using video playback of social agents to mimic real-life situations when investigating corvid decision making. 


\section{Difficulties when using video playback to investigate social cognition in}

2 California scrub-jays (Aphelocoma californica)

3 Katharina F. Brecht ${ }^{1,2 *}$, Ljerka Ostojić ${ }^{1}$, Edward W. Legg ${ }^{1} \&$ Nicola S. Clayton ${ }^{1}$

$4 \quad{ }^{1}$ Department of Psychology, University of Cambridge, Cambridge, UK

$5 \quad{ }^{2}$ Institute of Neurobiology, University of Tübingen, Tübingen, Germany

6 * Corresponding author: Katharina Brecht

7 Email address: katharinabrecht@gmail.com

\section{ABSTRACT}

9 Previous research has suggested that videos can be used to experimentally manipulate social 10 stimuli. In the present study, we used the California scrub-jays' cache protection strategies to 11 assess whether video playback can be used to simulate conspecifics in a social context. In both 12 the lab and the field, scrub-jays are known to exhibit a range of behaviours to protect their caches 13 from potential pilferage by a conspecific, for example by hiding food in locations out of the 14 observer's view or by re-caching previously made caches once the observer has left. Here, we 15 presented scrub-jays with videos of a conspecific observer as well as two non-social conditions 16 during a caching period and assessed whether they would cache out of the observer's "view" 17 (Experiment 1) or would re-cache their caches once the observer was no longer present 18 (Experiment 2). In contrast to previous studies using live observers, the scrub-jays' caching and 19 re-caching behaviour was not influenced by whether the observer was present or absent. These 20 findings suggest that there might be limitations in using video playback of social agents to mimic 21 real-life situations when investigating corvid decision making. 


\section{INTRODUCTION}

24 The use of video stimuli offers a high degree of flexibility in the presentation of stimuli in animal 25 experiments as it allows for a controlled presentation of inanimate objects as well as conspecifics 26 and social agents in general. Furthermore, the possibility to alter certain features allows the 27 experimenter to create well-suited control stimuli (D’Eath, 1998; Rieucau \& Giraldeau, 2009).

28 This characteristic of video clips can be especially helpful when assessing socio-cognitive 29 processes in animals. Here, an important advantage of using video over live stimuli is that video clips allow contrasting social and non-social stimuli congruently. Moreover, it is possible to present the same stimuli repeatedly or to different subjects, whereas presenting live conspecifics (or other social agents) is difficult to control in such situations. A recent example of the application of videos is a study by Sliwa and Freiwald (2017) assessing neuronal networks of social interactions in rhesus macaques. To unravel which brain regions were specifically dedicated for social interactions, fMRI scans were made of four macaques watching videos of conspecifics interacting together and contrasted to videos of an individual conspecific acting alone as well as with objects moving and interacting (and additional controls). Additionally, the use of video playback allowed for the precise control of the timing of the presentation of stimuli, which ensured average activations could be calculated across the subjects (Sliwa \& Freiwald, 2017). Hence, videos allow for a controlled investigating of social cognition.

Birds of the crow family are well-known for their socio-cognitive abilities (e.g., Clayton \& Emery, 2015). Here too, videos could provide a useful means for manipulating and displaying social stimuli to investigate their socio-cognitive abilities in more detail, for example, in order to assess which cues elicited by a conspecific the birds are sensitive to. However, the suitability of video playback in behavioural experiments with birds in general has been contested (D'Eath, 1998). There are a suite of limitations regarding the presentation of video stimuli to birds, stemming from the fact that video screens are built for the human not the avian visual system (D'Eath, 1998; Lea \& Dittrich, 2000). One example is the critical flicker-fusion frequency (CFF): The continuous perception of individual screens as a video differs between animals, with humans perceiving a video with 25 frames per second as continuous, while for example zebra finches have a CFF of $55.3 \mathrm{~Hz}$ (Crozier \& Wolf, 1941) and therefore may perceive the same video as flickering as opposed to continual, seamless motion. While there are studies confirming that these limitations pose a problem for the suitability of video playback with birds (D'Eath \& 
54 Dawkins, 1996; Ryan \& Lea, 1994), there are also reports of successful use to display conspecifics to birds in different contexts. For example, in chickens, video playback of conspecifics produces audience effects (Evans \& Marler, 1991) and affects feeding behaviour via social facilitation (Keeling \& Hurnik, 1993). Male Zebra finches react appropriately to the display of a female conspecific, even to subtle changes in behaviour of the female (Galoch \& Bischof, 2007) and use the feeding choice of a demonstrator bird presented as video playback to guide their own feeding decision (Guillette \& Healy, 2017). Video playback of a group of conspecifics produced similar effects on foraging in nutmeg mannikins to when real conspecifics were present (Rieucau \& Giraldeau, 2009). Additional examples include studies in starlings (Zoratto et al., 2014), quail (Ophir \& Galef, 2003) and junglefowl (McQuoid \& Galef, 1993). In corvids in particular, a recent study reported that Eurasian jays were able to transfer learned associations about live objects to videos of these objects (Davidson, Miller, Loissel, Cheke, \& Clayton, 2017). However, this study involved only inanimate objects. In the social domain, Bird and Emery (2008) showed that rooks prefer observing their partner over an unfamiliar conspecific, both when presented live and as video playback. Similar results have also been obtained with California scrub-jays (Brecht, 2017). However, it remains unclear whether the birds perceived conspecifics displayed as video playback as relevant social stimuli (i.e., as their partner).

While the rooks showed the same preference in the video and the live conditions, it is possible that they looked more at the video of the partner because they saw certain cues associated with their partner and a preference for these cues over other cues may be possible even if the birds did not interpret the bird in the video as their partner. Additionally, while birds seem to be sensitive to what is presented, it is not known to what extent they make use of this information. For example, while blue tits seem to be responsive to a conspecific presented on screen, it is less clear whether they use the social information provided by this conspecific (Hämäläinen, Rowland, Mappes, \& Thorogood, 2017). Studies showing bird species changing their mating or feeding behaviour (e.g., McQuoid \& Galef, 1993; Rieucau \& Giraldeau, 2009) in response to a video of conspecifics could be due to the perception of certain cues that reflexively trigger such responses, similar to an artificial red stimulus that is sufficient in eliciting begging behaviour in herring gulls (Tinbergen \& Perdeck, 1950). Recognising conspecifics as such does not imply that they are also interpreted as relevant for the current behaviour. Consequently, in 
85 order to use video playback as a means to study social cognition in the avian taxa, it is necessary

86 to investigate whether birds, and corvids in particular, perceive conspecifics presented as video

87 playback as relevant social stimuli that impact the subjects' behaviours and decision-making.

88 In order to assess this question, we used the scrub-jays' propensity to protect their caches

89 from their conspecifics. Like other corvids, scrub-jays cache excess food for later consumption

90 (Vander Wall, 1990). This cached food is at risk of being pilfered by conspecifics, whose

91 observational spatial memory allows them to efficiently pilfer caches they have seen others make

92 (Watanabe \& Clayton, 2007). To protect their caches from being pilfered by a conspecific

93 observer, scrub-jays have been found to employ a suite of different behavioural strategies (Dally,

94 Clayton, \& Emery, 2006; Grodzinski \& Clayton, 2010). Specifically, when observed by a

95 conspecific, scrub-jays preferentially cache in locations that limit the observer's visual access to

96 the cache site, such as in the shade, behind barriers, or far away from the observer (Dally, Emery,

97 \& Clayton, 2005). If they cannot obscure the location of their caches from the observer at the

98 time of caching (Dally et al., 2005), scrub-jays move previously made caches to different, novel

99 locations (re-caching) once the potential threat has left the scene (Dally, Emery, \& Clayton,

100 2006; Emery \& Clayton, 2001). Similar behaviours have been reported for other corvid species,

101 namely ravens (e.g., Bugnyar, 2011; Bugnyar \& Heinrich, 2005), Clark’s nutcrackers (Clary \&

102 Kelly, 2011) and Eurasian jays (e.g., Legg \& Clayton, 2014; Shaw \& Clayton, 2012).

103 Importantly, such strategies are employed in response to social cues (e.g., Clary \& Kelly, 2011),

104 and seem to be specific to situations in which caching is observed by a conspecific competitor

105 (e.g., Dally et al., 2010; Thom \& Clayton, 2013).

106 In two experiments, we presented scrub-jays with video playback of a conspecific

107 observer while they had the opportunity to cache. We assessed two different cache-protection

108 strategies: caching out of the observer's view (Experiment 1) and re-caching once the observer

109 has left (Experiment 2). If scrub-jays interpreted the conspecific observer in the video as a threat

110 to their caches - like they do with live conspecific observers - they should selectively protect

111 their caches, i.e. preferentially cache out-of-view (Experiment 1) and re-cache (Experiment 2)

112 when the video showed a conspecific than when the video showed an empty cage or a rope

113 hanging from the cage ceiling (non-social conditions). 


\section{EXPERIMENT 1}

115 In Experiment 1, scrub-jays were presented with videos of an observer (Observer

116 condition). In addition, we tested the birds in two non-social conditions: in one, the birds were

117 presented with a video of an empty cage (Empty Cage condition) and in the second condition,

118 birds were presented with a video of a lightly swinging rope hanging from the cage ceiling (Rope

119 condition). This second non-social condition was run to ascertain that a difference between the

120 Observer and the Empty Cage condition would not be due to movement on the screen.

121 While they were presented with one of the three videos, scrub-jays could cache food in

122 two different locations - one "in-view" of the video screen and one "out-of-view", behind an

123 opaque barrier. The set-up was similar to methods previously used to assess cache-protection

124 strategies in scrub-jays, in that scrub-jays were able to choose between a caching site in-view and

125 a caching site out-of-view of the observer (e.g., Dally et al., 2010). If birds are responding to

126 video playback of the conspecific similarly to how they respond to live observers (reviewed in

127 Clayton et al., 2007; Grodzinski \& Clayton, 2010), they should cache more in the "out-of-view"

128 than in the "in-view" location when presented with the video of the conspecific observer.

129 Alternatively, if the birds showed no difference in their caching behaviour between the observer

130 and the non-social conditions, this would suggest that - unlike in a 'live' situation - they did not

131 perceive the conspecific in the video as a threat to their caches.

\section{Method}

133 Subjects. Five female and five male adult scrub-jays were tested between December

1342014 and February 2015. Scrub-jays were housed at the Sub-department of Animal Behaviour,

135 University of Cambridge, between May and August 2015. The work was conducted under the

136 UK Home Office project licence PPL 80/2519. During the experimental period, subjects were

137 housed in pairs in three to four joined cages (each measuring 1 x 1 x $1 \mathrm{~m}$ ). Outside of testing

138 birds rested in outside aviaries.

139 Procedure. In a within-subject design, each bird participated in all of the three conditions

140 and completed three separate test trials on three consecutive days. Hence, each bird saw each

141 video once. Trial order was pseudo-randomised across birds.

142 Individuals could either cache in a location hidden behind the opaque side of a Perspex

143 barrier ("out-of-view" location) or behind a transparent side of a Perspex barrier ("in-view" 
144 location). Which side of the barrier was opaque was counterbalanced across subjects but held 145 constant across conditions.

146 Two hours prior to testing, individuals were separated from their cage partner, and

147 maintenance diet was removed to ensure that birds were mildly hungry and thus motivated to

148 cache or eat at the time of testing. Birds were tested in cage 1 (see Figure 1, Panel A). At the start

149 of a trial, dividers to cage 2 were removed and the screen, mounted onto the back wall, was

150 showing one of the three videos. Birds were given a 15-minute long test phase during which they

151 could cache. Birds were then released into their home cage and caching trays were removed for a

152 15-minute long break. During this break, caches were counted and placed back in the tray. Any

153 caches made outside the tray were removed. Finally, birds were again allowed into cage 1 to

154 retrieve any caches made previously, a procedure that has been successfully used to prevent

155 extinction of caching in previous caching studies (reviewed in Clayton et al., 2007). During this

156 15-minute long retrieval phase, the screen was covered. Subsequently, the caching trays were

157 removed, birds were released into their home cage and maintenance diet was returned.

158 In a pre-test, two trials were conducted to ensure that birds were comfortable with

159 caching in the experimental set-up, particularly in the presence of the computer screen. In these

160 pre-test trials, birds were given access to a bowl containing food items and a single caching tray,

161 which was placed in one trial behind the opaque, and in another trial behind the transparent side

162 of the Perspex barrier. Birds were allowed to cache for 15 minutes whilst the video on the

163 computer screen showed an empty cage. The order in which the birds experienced the caching

164 tray being behind the opaque or the transparent sides of the barrier was counterbalanced across

165 birds. Trials were repeated until each bird cached in both trays, with a maximum of two trials per 166 side.

167 Material. The videos presented were 15-minute long, without sound, and consisted of a 168 looped sequence of 4 to 5-second long recordings. Videos were filmed using a GoPro® Hero 4

169 Black with 60 frames per second and a resolution of 930p and presented with a Lenovo®

170 Thinkpad Edge E330 (50 to $60 \mathrm{~Hz}$ refresh rate, graphic card Intel HD graphics 4000) on a 17”

171 portable Lenovo ${ }^{\circledR}$ LCD monitor $(60 \mathrm{~Hz}$ refresh rate). Note that LCD pixels do not flash between

172 frames, and hence, LCD monitors exhibit no refresh-induced flicker.

173 The screen with the video was mounted to the wall of cage 2 (see Figure 1, Panel A). The

174 videos presented either a conspecific facing the cacher (Observer condition), an empty cage 
175 (Empty Cage condition), or a rope hanging from the ceiling (Rope condition; see Figure 1, Panel

$176 \mathrm{C})$. On screen, conspecifics were between $6.4 \mathrm{~cm}$ to $8 \mathrm{~cm}$ in height, corresponding to a visual

177 angle of $3.3^{\circ}$ to $4.2^{\circ}$ (assuming the cacher stands on the caching tray).

178 Birds serving as actors were kept mildly hungry by removing all food from their cage 30

179 minutes prior to filming. For filming the Observer condition, actor birds were presented with

180 food behind the camera to ensure that they attended to the direction of the camera and thus

181 would appear to be attending to the cacher during the test trials.

182 Actors were chosen based on their reaction to being filmed: we choose only birds that sat

183 calmly on a perch in front of the camera. All actor birds were familiar to the focal birds and were

184 of the same sex as the respective focal bird. Three sets of videos were shot with three different

185 birds to prevent any effects being caused by study-irrelevant features of the video, such as the

186 identity of the conspecific shown.

187

188

--- Figure 1 ---

189

190

191

192

193 one of the two locations ("in view" versus "out-of-view") in Experiment 1, the difference of the number of caches made in the "out-of-view" tray minus the number of caches made in the "inview tray" $\left(\mathrm{D}=\mathrm{Cached}_{\text {out-of-view }}-\mathrm{Cached}_{\text {in view }}\right)$ was compared across conditions. If scrub-jays represented the conspecific observer in the video as a threat to their caches, this difference should be larger in the Observer condition than in the two non-social conditions, $\mathrm{D}_{\text {Observer }}>$ $\mathrm{D}_{\text {Empty Cage }}$ and $\mathrm{D}_{\text {Observer }}>\mathrm{D}_{\text {Rope }}$.

Aligned rank transformed data (Wobbrock, Findlater, Gergle, \& Higgins, 2011) were submitted to a repeated measures ANOVA with the within-subject factor Condition (Empty Cage vs. Observer vs. Rope). Alpha was set at .05. One-sided permutation tests were calculated using 
205 R package coin (Hothorn, Hornik, van de Wiel, \& Zeileis, 2008) as a planned contrast between

206

207

208

209

210

211

212

213

214

215

216

217

218

219

220

221

222

223

224

225

226

227

228

229

230

231

232

233

234 the Observer condition and non-social conditions (Empty Cage and Rope, separately).

Additionally, we analysed the data using Bayesian statistics. A failure to reject the nullhypothesis using frequentist inferences cannot be used to draw conclusions about the absence of an effect (Dienes, 2016). Thus, we calculated a Bayes Factor $(B F)$ using JASP Version 0.8.1.2 (JASP Team, 2016) with a Bayesian repeated measures ANOVA with the within-subject factor Condition (Empty Cage vs. Observer vs. Rope). Here, the null hypothesis being that the

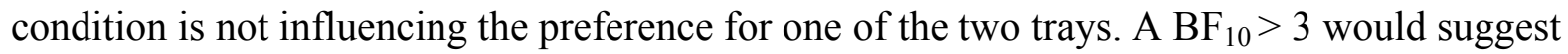
support for $\mathrm{H} 1$ (i.e., $\mathrm{D}_{\text {Observer }}>\mathrm{D}_{\text {Empty Cage }}$ and $\mathrm{D}_{\text {Observer }}>\mathrm{D}_{\text {Rope }}$ ) and a $\mathrm{BF}_{01}<0.333$ would suggest support for the $\mathrm{H} 0$ (i.e., $\mathrm{D}_{\text {Observer }}=\mathrm{D}_{\text {Empty Cage }}$ and $\mathrm{D}_{\text {Observer }}=\mathrm{D}_{\text {Rope }}$ ).

\section{Results}

One male subject (Subject No. 210) was excluded from the analysis because he failed to cache for the duration of all test trials (final $n=9$ ). Table 1 shows the median number of caches made in both trays. There was no main effect of Condition on the total number of caches made $(F(1,16)=1.34, p=.290)$.

---- Table 1 ----

Critically, there was also no main effect of Condition on the difference of caches made in the "in-view" minus "out-of-view" tray $(F(2,16)=0.572, p=.575$; see Figure 2). Planned contrasts showed that the difference of caches made in the "out-of-view" tray minus the "inview" tray in the Observer condition (Median = -1 , range $=21$ ) was not higher than in the Empty Cage condition (Median $=-3$, range $=9 ; Z=-0.043, p=.523, d=-0.014$ ), or the Rope condition $($ median $=0.5$, range $=19.5 ; Z=-1.003, p=.838, d=-0.337)$.

\section{--- Figure 2 ----}

(1)

In order to check whether the trial sequence had an effect on caching, we assessed the number of caches made in both trays across trials post-hoc. Table 1, lower part, shows the number of caches made per condition. There was no difference in number of caches made $(F(2,16)=0.021, p=.980)$. 
The Bayes factor was $B F_{10}=0.279 \pm 0.572$, suggesting that our data support the null-

236 hypothesis, i.e., a similar preference for the two trays across all three conditions. Thus, our data

237 suggest that the scrub-jays did not adjust their cache-protection strategies in response to the

238 video playback presented. When comparing the Empty Cage and the Observer condition with a

239 Bayesian t-test, we found again that our data supported the null-hypothesis, $\mathrm{D}_{\text {Observer }}=\mathrm{D}_{\text {Empty Cage, }}$

$240 B F_{10}=0.331 \pm \sim 0.004$, whilst the comparison between the Rope and the Observer conditions

241 showed only anecdotal evidence for the null-hypothesis, $\mathrm{D}_{\text {Observer }}=\mathrm{D}_{\text {Rope }}, B F_{10}=0.780 \pm$

$242 \sim 1.560 \mathrm{e}^{-4}$.

243 These findings suggest that the scrub-jays' caching behaviour was not affected by the

244 presence of the conspecific displayed on the video screen: the birds were not more likely to

245 employ cache protection strategies when presented with a video of a conspecific compared to the

246 non-social controls.

\section{EXPERIMENT 2}

248 Experiment 2 addressed the question of whether the jays' re-caching behaviour was

249 influenced by the different videos. Cachers had access to two caching trays, both "in-view", and,

250 after a break of 15 minutes, had the opportunity to re-cache 'in private', i.e., in the absence of the

251 observer, any caches made in the initial caching period. As in Experiment 1, birds were

252 presented with different videos during caching, namely of an observer (social condition), as well

253 as of an empty cage and a rope hanging from the ceiling (non-social conditions). The proportion

254 of re-caches (number of re-caches divided by the total number of caches) made was investigated.

255 If the scrub-jays were sensitive to the video presented on the video screen, they were expected to

256 re-cache a larger proportion of initially made caches after having had cached in front of a video

257 showing the observer (Observer condition) than after having had cached in front of a video

258 showing an empty cage (Empty Cage condition) or a rope hanging from the ceiling (Rope

259 condition).

260 Method

261 Subjects. Five female and four male ${ }^{1}$ sexually mature scrub-jays were housed and tested

262 under the same conditions as laid out in Experiment 1 between February and June 2015. All

${ }^{1}$ One male bird, 210, was not tested because he failed to cache in Experiment 1. 
263 birds had previously participated in Experiment 1. The overall setup was the same as in

264 Experiment 1, except that a fully transparent divider was placed between cages 1 and 2 such that

265 there was no difference in the visual accessibility of the two caching locations, i.e., both caching 266 trays were "in-view".

267 Procedure and Material. Each bird completed three test trials, separated into 3 phases,

268 as described in Experiment 1. Importantly, during the 15-minute long re-caching phase, the video

269 screen was covered, thus re-caching was in "private". Note that new videos were shot for this

270 experiment to avoid habituation, and new actor birds were used, that is, the focal birds saw a

271 different observer than in Experiment 1.

272 Analysis. To ensure that birds could re-cache items, birds had to initially have cached at

273 least one food item in each condition. In an equivalent manner to Experiment 1, we analysed the

274 proportion of re-cached items (number of re-cached items divided by total number of items

275 cached) with a repeated measures ANOVA (factor Condition) and one-sided permutation tests as

276 planned contrasts.

277 Additionally, we calculated a Bayes Factor $(B F)$ using JASP Version 0.8.1.2 (JASP

278 Team, 2016) with a Bayesian repeated measures ANOVA with the within-subject factor

279 Condition (Empty Cage vs. Observer vs. Rope). Here, the null hypothesis being that the

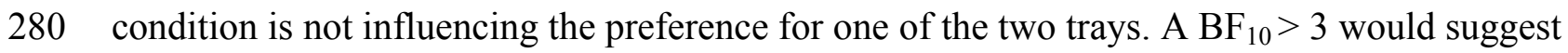

281 support for $\mathrm{H} 1$ and a $\mathrm{BF}_{01}<0.333$ would suggest support for the $\mathrm{H} 0$.

282 Results

283 Subject Nr. 215 was excluded from the analysis because she failed to cache across all test 284 trials (final $n=8$ ). Table 2 shows the median number of caches made and the proportion of re285 cached items. There was no main effect of Condition on the number of caches made in the 286 caching phase $(F(1,14)=1.133, p=.349)$.

287

288

---- Table 2 ----

289

290 Crucially, there was no effect of Condition on re-caching, that is, birds did not alter the

291 proportion of re-cached items depending on the different stimuli presented on the video, $(F(2,14)$

$292=0.29, p=.75$, see Figure 3$)$. Birds did not re-cache a larger proportion of caches in the

293 Observer condition (Median $=0.44$, Range 0.31-0.62) than in the Empty Cage condition (Median 
$294=0.37$, range: $0.22-1 ; Z=-0.041, p=.520, d=0.00)$, or than in the Rope condition

$295($ Median $=0.42$, range: $0.29-1 ; Z=-0.371, p=.582, d=0.105)$.

296 Similarly to Experiment 1, we assessed whether the trial sequence had an effect on 297 caching as well as on the proportion of re-caching. Table 2, lower part, shows the number of 298 cached and re-cached items per trial number. There was no effect of trial number on the 299 proportion of re-cached items $(F(2,14)=0.009, p=.991)$. There was also no effect of trial 300 number on the number of cached items $(\mathrm{F}(2,14)=0.404, \mathrm{p}=.675)$.

301

302

--- Figure 3 ---

303

304

305

The Bayes factor was $B F_{10}=0.282 \pm 0.780$, suggesting that our data support the nullhypothesis, i.e., the amount of re-caches was similar across conditions. Comparisons using Bayesian t-tests showed that there was no difference in re-caches between the Observer and the Empty Cage conditions, $B F_{10}=0.327 \pm \sim 0.014$ or the Observer and the Rope conditions, $B F_{10}=$ $0.268 \pm \sim 0.002$. Thus, our results suggest that the scrub-jays did not adjust their re-caching behaviour in response to the video playback presented.

\section{GENERAL DISCUSSION}

In this study, we used California scrub-jays' cache protection strategies to assess the use

312 of video playback when presenting social stimuli to corvids. Scrub-jays are known to protect

313 their caches against potential pilferage by conspecific observers by caching out of the

314 conspecific's view (Clayton et al., 2007; Dally et al., 2010) or by re-caching previously made

315 caches (Emery \& Clayton, 2001). When presented with the video playback of a conspecific,

316 scrub-jays did not employ protection strategies in either their caching or re-caching behaviours,

317 suggesting that they do not respond to video playback in the same way they respond to live 318 observers.

319 More specifically, in Experiment 1, the scrub-jays did not prefer to cache in an "out-of-

320 view" tray over an "in-view" tray when presented with a video playback of a conspecific

321 compared to the two non-social conditions. Similarly, in Experiment 2, the birds did not re-cache

322 more when presented with a video of conspecific during an initial caching event compared to the

323 non-social conditions. In a suite of previous studies using live observers with a very similar

324 setup, the scrub-jays cached more in sites that were "out-of-view" of an observer and re-cached 
325 their caches once an observer has left the scene, while they did not show the same pattern of

326 behaviours in a non-social condition, i.e., when they were caching in private (Dally et al., 2005,

327 2010; Emery \& Clayton, 2001; Thom \& Clayton, 2013). Although null results are difficult to

328 interpret, our study suggests that the birds may not respond to video playback conditions in the

329 same way as when confronted with a live conspecific in the context of caching. It should be

330 noted that during the pre-test phase, the birds were shown an empty cage, albeit not the same one

331 as in the test. However, familiarity with the empty cage video could explain why birds cached

332 more in the empty cage condition (although this difference was not significant) in Experiment 1.

333 Consequently, contrary to the reports of successful use of video playback to display test

334 stimuli to birds (e.g, Galoch \& Bischof, 2007; McQuoid \& Galef, 1993; Ophir \& Galef, 2003;

335 Zoratto et al., 2014), the birds in the present study did not adjust their behaviour to what was

336 shown on the video. Especially relevant for this present study is the finding that rooks are able to

337 differentiate between individual conspecifics presented on a video screen (Bird \& Emery, 2008).

338 In addition, we have previously shown that scrub-jays prefer to look at their conspecific partner

339 compared to an object, regardless of whether the conspecific was presented as video or live

340 (Brecht, 2017). Hence, our results represent a noteworthy divergence from previous studies,

341 considering that we provided the birds with videos similar to those successfully used in previous

342 video playback experiments (e.g., Bird \& Emery, 2008; Guillette \& Healy, 2017). There are

343 several cues that might be relevant in social interactions besides visual displays that were not

344 present in the current study, such as olfactory and auditory cues, which, in real-life situations,

345 can be used to recognise conspecifics (D’Eath \& Dawkins, 1996). Compared to other bird

346 species, however, corvids have a small olfactory bulb (Bang, 1971; Healy \& Guilford, 1990) and

347 are seen to use their sense of smell predominantly for detecting food (e.g., Buitron \&

348 Nuechterlein, 1985; Harriman \& Berger, 1986). Additionally, there is so far no evidence that

349 olfactory cues play a marked role in the social cognition of corvids. Hence, while it remains a

350 possibility, given our current knowledge, it seems unlikely that the lack of olfactory cues might

351 be responsible for the failure of the birds to adjust their behaviour to the video displays. In

352 contrast to olfactory cues, auditory cues have been found to be of relevance when presenting

353 video playback to birds (e.g., Galoch \& Bischof, 2007). In other studies however, auditory cues

354 were not necessary to prompt social learning (Guillette \& Healy, 2017). In future studies, it will 
355 be important to manipulate both visual and acoustic cues when using video playback to

356 investigate the role of each cue separately and of both cues together.

357 In our case, it seems likely that the absence of cache-protection strategies reported is

358 associated with factors that might be especially important for the socio-cognitive nature of the

359 caching context. One potentially important feature of a conspecific that was not available to the

360 birds in this study specifically might be a real time response in the behaviour of the video

361 conspecific. The relevance of a conspecific's behaviour has previously been reported in the

362 successful recognition of and discrimination between conspecifics (Keeling \& Hurnik, 1993;

363 McQuoid \& Galef, 1993; Shimizu, 1998). Similarly, it has been proposed that an absence of

364 interaction with the focal bird seems to deter hens from treating video stimuli as real conspecifics

365 (D'Eath \& Dawkins, 1996). Hence, the indifference towards the observer could have been be due

366 to the lack of behaviours from the video-observer: In Experiments 1 and 2, looped material was

367 used in order to present the birds with a conspecific being in view and attentive throughout the

368 whole video (as opposed to sleeping for example), which might have created an unusual

369 deficiency of dynamic motion. In reality, it might be rare that a conspecific is facing towards a

370 conspecific for a prolonged amount of time and does not interact with the cacher. Consequently,

371 the altered video playback of conspecifics used here might not have been sufficient to elicit

372 cache-protection strategies due to what they depicted. Hence, in order to guide complex decision

373 making, such as a bird's caching behaviour, further research might need to include unaltered

374 video playback.

375 While not the main focus of the present study, our results raise the additional question of

376 which features of an observer trigger cache-protection strategies in corvids. One possibility

377 might be the 'interaction' with the conspecific. In contrast to the dynamic relationship between a

378 cacher and a live conspecific observer, the conspecific in the video did not respond to the

379 cacher's behaviour in any way. This lack of interaction may have been an unusual situation for

380 the birds being tested and might have led them to believe that their caches were safe, or at least

381 that circumstances were "unusual". On a related note, scrub-jays have been shown to treat a

382 mirror-image of themselves during caching as if they were in private, rather than as if the

383 observed image in the mirror was a live observer. In other words, the 'conspecific' reflected by

384 the mirror image was not sufficient to elicit cache protection strategies (Dally et al., 2010). In

385 addition, a cache-protection strategy that takes into account the current motivational state of an 
386 observer seems to be based on the behaviour of the observer at the time of caching (Ostojić et al.,

387 2017). Consequently, it might be the contingent responses from an interactant that could serve as

388 a salient cue that the birds use to employ cache protection strategies. Throughout their lives, the

389 scrub-jays might have learned that conspecifics will respond to their caching behaviour, for

390 example by coming closer to the caching site or by carefully monitoring the caching. Hence,

391 further research is needed to assess which aspects of the behaviour of an observer are necessary

392 conditions for cache-protection strategies to occur (Ostojić et al., 2017).

\section{Conclusion}

394 In conclusion, our results suggest limitations in using video playback to present social stimuli to

395 corvids. Depending on the context, different stimuli of a conspecific might be necessary in order

396 to recognise conspecifics, and to behave accordingly. Specifically, there might be some

397 limitations in regards to the use of video playback to mimic real-life situations involving social

398 agents, such as being observed by a conspecific, to a sufficient extent to influence the birds'

399 decision making such as their cache-protection behaviour.

400 ACKNOWLEDGEMENTS

401 We thank Sam Melvin, Sarah Manley, and Ivan Vakrilov for animal husbandry. We thank two

402 anonymous reviewers for helpful comments on a previous version of the manuscript. 


\section{REFERENCES}

405 Bang, B. G. 1971. Functional anatomy of the olfactory system in 23 orders of birds. Acta 406 Anatomia, Suppl. 58:1-76.

407 Bird, C. D., \& Emery, N. J. 2008. Using video playback to investigate the social preferences of 408 rooks, Corvus frugilegus. Animal Behaviour, 76:679-687. DOI:

$409 \quad$ 10.1016/j.anbehav.2008.04.014

410 Brecht, K. F. 2017. A multi-facetted approach to investigating theory of mind in corvids. D. Phil. 411 Thesis, University of Cambridge. DOI: 10.17863/CAM.12978

412 Bugnyar, T. 2011. Knower-guesser differentiation in ravens: others' viewpoints matter.

413 Proceedings of the Royal Society of London B: Biological Sciences, 278:634-640. DOI:

$414 \quad 10.1098 /$ rspb.2010.1514

415 Bugnyar, T., \& Heinrich, B. 2005. Ravens, Corvus corax, differentiate between knowledgeable

416 and ignorant competitors. Proceedings of the Royal Society of London B: Biological

417 Sciences, 272:1641-6. DOI: 10.1098/rspb.2005.3144

418 Buitron, D., \& Nuechterlein, G. L. 1985. Experiments on olfactory detection of food caches by 419 black-billed magpies. The Condor, 87:92-95. DOI: 10.2307/1367139

420 Clary, D., \& Kelly, D. M. 2011. Cache protection strategies of a non-social food-caching corvid, 421 Clark's nutcracker (Nucifraga columbiana). Animal Cognition, 14:735-44. DOI:

$422 \quad 10.1007 / \mathrm{s} 10071-011-0408-3$

423 Clayton, N. S., Dally, J. M., \& Emery, N. J. 2007. Social cognition by food-caching corvids. The 424 Western scrub-jay as a natural psychologist. Philosophical Transactions of the Royal 425 Society B, 362:507-22. DOI: 10.1098/rstb.2006.1992

426 Clayton, N. S., \& Emery, N. J. 2015. Avian Models for Human Cognitive Neuroscience: A 427 Proposal. Neuron, 86:1330-1342. DOI: 10.1016/j.neuron.2015.04.024

428 Crozier, W. J., \& Wolf, E. 1941. Thee simple flicker threshold contour for the zebra finch. The 429 Journal of General Physiology, 24:625-633. DOI: 10.1085/jgp.24.5.625

430 D’Eath, R. B. 1998. Can video images imitate real stimuli in animal behaviour experiments?

431 Biological Reviews, 73:267-292. DOI: 10.1111/j.1469-185X.1998.tb00031.x

432 D'Eath, R. B., \& Dawkins, M. S. 1996. Laying hens do not discriminate between video images

433 of conspecifics. Animal Behaviour, 52:903-912. DOI: 10.1006/anbe.1996.0238

434 Dally, J. M., Clayton, N. S., \& Emery, N. J. 2006. The behaviour and evolution of cache 
435

436

437

438

439

440

441

442

443

444

445

446

447

448

449

450

451

452

453

454

455

456

457

458

459

460

461

462

463

464

465

protection and pilferage. Animal Behaviour, 72:13-23. DOI:

10.1016/j.anbehav.2005.08.020

Dally, J. M., Emery, N. J., \& Clayton, N. S. 2005. Cache protection strategies by western scrubjays, Aphelocoma californica: implications for social cognition. Animal Behaviour, 70:1251-1263. DOI: 10.1016/j.anbehav.2005.02.009

Dally, J. M., Emery, N. J., \& Clayton, N. S. 2006. Food-caching Western scrub-jays keep track of who was watching when. Science, 312:1662-1666. DOI: 10.1126/science.1126539

Dally, J. M., Emery, N. J., \& Clayton, N. S. 2010. Avian Theory of Mind and counter espionage by food-caching Western scrub-jays (Aphelocoma californica). European Journal of Developmental Psychology, 7:17-37. DOI: 10.1080/17405620802571711

Davidson, G., Miller, R., Loissel, E., Cheke, L. G., \& Clayton, N. S. 2017. The development of support intuitions and object causality in juvenile Eurasian jays (Garrulus glandarius). Scientific Reports, 7:40062. DOI: 10.1038/srep40062

Dienes, Z. 2016. How Bayes factors change scientific practice. Journal of Mathematical Psychology, 72:78-89. DOI: 10.1016/j.jmp.2015.10.003

Emery, N. J., \& Clayton, N. S. 2001. Effects of experience and social context on prospective caching strategies by scrub jays. Nature, 414:443-446. DOI: 10.1038/35106560

Evans, C. S., \& Marler, P. 1991. On the use of video images as social stimuli in birds: audience effects on alarm calling. Animal Behaviour, 41:17-26. DOI: 10.1016/S00033472(05)80499-3

Galoch, Z., \& Bischof, H.-J. 2007. Behavioural responses to video playbacks by zebra finch males. Behavioural Processes, 74:21-26. DOI: 10.1016/j.beproc.2006.09.002

Grodzinski, U., \& Clayton, N. S. 2010. Problems faced by food-caching corvids and the evolution of cognitive solutions. Philosophical Transactions of the Royal Society of London. Series B, Biological Sciences, 365:977-87. DOI: 10.1098/rstb.2009.0210

Guillette, L. M., \& Healy, S. D. 2017. The roles of vocal and visual interactions in social learning zebra finches: A video playback experiment. Behavioural Processes, 139:43-49. DOI: 10.1016/j.beproc.2016.12.009.

Hämäläinen, L., Rowland, H. M., Mappes, J., Thorogood, R. 2017. Can video playback provide social informtion for foraging blue tits? PeerJ, 5:e3062. DOI: 10.7717/peerj.3062

Harriman, A. E., \& Berger, R. H. 1986. Olfactory acuity in the common raven (Corvus corax). 
Healy S., \& Guilford T. 1990 Olfactory-Bulb Size and Nocturnality in Birds. Evolution, 44:339346. DOI: $10.2307 / 2409412$

Hothorn, T., Hornik, K., van de Wiel, M. A., \& Zeileis, A. 2008. Implementing a class of permutation tests: The coin package. Journal of Statistical Software, 28:1-23.

Keeling, L. J., \& Hurnik, J. F. 1993. Chickens show socially facilitated feeding behaviour in response to a video image of a conspecific. Applied Animal Behaviour Science, 36:223-231. DOI: 10.1016/0168-1591(93)90012-E

Lea, S. E. G., \& Dittrich, W. H. 2000. What do birds see in moving video images? In J. Fagot, ed., Picture Perception in Animals. Hove: Psychology Press, 143-180.

Legg, E. W., \& Clayton, N. S. 2014. Eurasian jays (Garrulus glandarius) conceal caches from onlookers. Animal Cognition, 17:1223-1226. DOI: 10.1007/s10071-014-0743-2

McQuoid, L. M., \& Galef, B. G. 1993. Social influences on feeding site selection by Burmese fowl (Gallus gallus). Journal of Comparative Psychology, 106:137-141. DOI: 10.1037/0735-7036.106.2.137

Ophir, A. G., \& Galef, B. G. 2003. Female Japanese quail that "eavesdrop" on fighting males prefer losers to winners. Animal Behaviour, 66:399-407. DOI: 10.1006/anbe.2003.2230

Ostojić, L., Legg, E. W., Brecht, K. F., Lange, F., Deininger, C., Mendl, M., \& Clayton, N. S. 2017. Current desires of conspecific observers affect cache-protection strategies in California scrub-jays and Eurasian jays. Current Biology, 27:R51-R53. DOI: 10.1016/j.cub.2016.11.020

Rieucau, G., \& Giraldeau, L.-A. 2009. Video playback and social foraging: simulated

Ryan, C. M. E., \& Lea, S. E. G. 1994. Images of conspecifics as categories to be discriminated by pigeons and chickens: Slides, video tapes, stuffed birds and live birds. Behavioural Processes, 33:155-175. DOI: 10.1016/0376-6357(94)90064-7 and pilfering tactics in response to social context. Animal Behaviour, 84:1191-1200. DOI: 10.1016/j.anbehav.2012.08.023

Shimizu, T. 1998. Conspecific recognition in pigeons (Columba livia) using dynamic video 
497

498

499

500

501

502

503

504

505

506

507

508

509

510

511

512

513

514

515

516

517

images. Behaviour, 135:43-53. DOI: 10.1163/156853998793066429

Sliwa, J., \& Freiwald, W. A. 2017. A dedicated network for social interaction processing in the primate brain. Science, 356: 745-749. DOI: 10.1126/science.aam6383

Tinbergen, N., \& Perdeck, A. C. 1950. On the Stimulus Situation Releasing the Begging Response in the Newly Hatched Herring Gull Chick (Larus Argentatus Argentatus Pont.). Behaviour, 3:1-39, 3:1-39.

Thom, J. M., \& Clayton, N. S. 2013. Re-caching by Western scrub-jays (Aphelocoma californica) cannot be attributed to stress. PloS One, 8:e52936. DOI: 10.1371/journal.pone.0052936

Vander Wall, S. B. 1990. Food hoarding in animals. Chicago: University of Chicago Press.

Watanabe, S., \& Clayton, N. S. 2007. Observational visuospatial encoding of the cache locations of others by western scrub-jays (Aphelocoma californica). Journal of Ethology, 25:271279. DOI: 10.1007/s10164-006-0023-y

Wobbrock, J. O., Findlater, L., Gergle, D., \& Higgins, J. J. 2011. The aligned rank transform for nonparametric factorial analyses using only ANOVA procedures. In Proceedings of the ACM Conference on Human Factors in Computing Systems (CHI' 11). Vancover, BC. New York, NY: ACM Press, 143-146. DOI: 10.1145/1978942.1978963

Zoratto, F., Manzari, L., Oddi, L., Pinxten, R., Eens, M., Santucci, D., ... Carere, C. 2014. Behavioural response of European starlings exposed to video playback of conspecific flocks: Effect of social context and predator threat. Behavioural Processes, 103:269-277. DOI: $10.1016 /$ j.beproc.2014.01.012 


\section{Figure 1 (on next page)}

Aerial view of the testing setups and example still frames of the videos used.

(A) An aerial view of the testing setup for Experiment 1. The cacher had access to two caching trays (red rectangle) and a bowl of food (orange circle). One tray was "out-of-view" behind the opaque barrier (thick grey line) and the other one was "in view" behind a transparent barrier (dotted grey line). The computer screen was mounted to the far wall of cage 2. (B) An aerial view of the testing setup for Experiment 2. Here, both trays are "inview" behind a transparent divider. (C) Still frames of the video clips, from the top: a conspecific facing towards the subject, an empty cage, and a rope hanging from the ceiling (non-social control). 

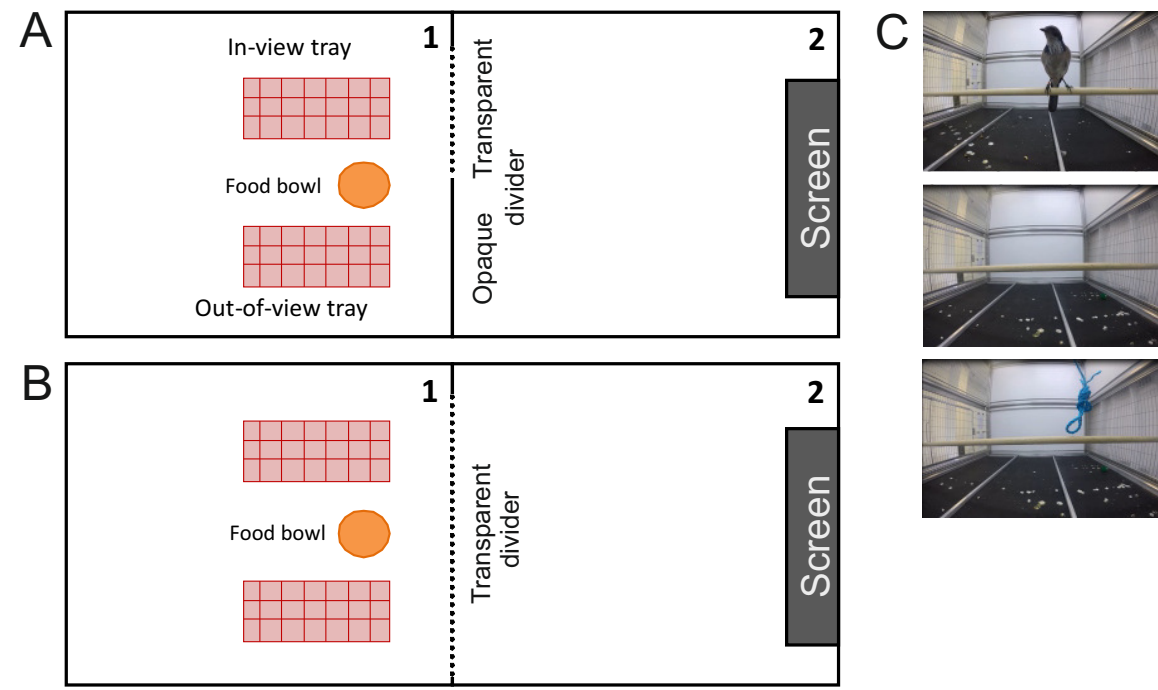
Figure 2 (on next page)

Number of caches cached in the "out-of-view" tray minus the number of caches cached in the "in-view" tray in Experiment 1.

Each data point indicates the difference for one bird in each of the three conditions. 


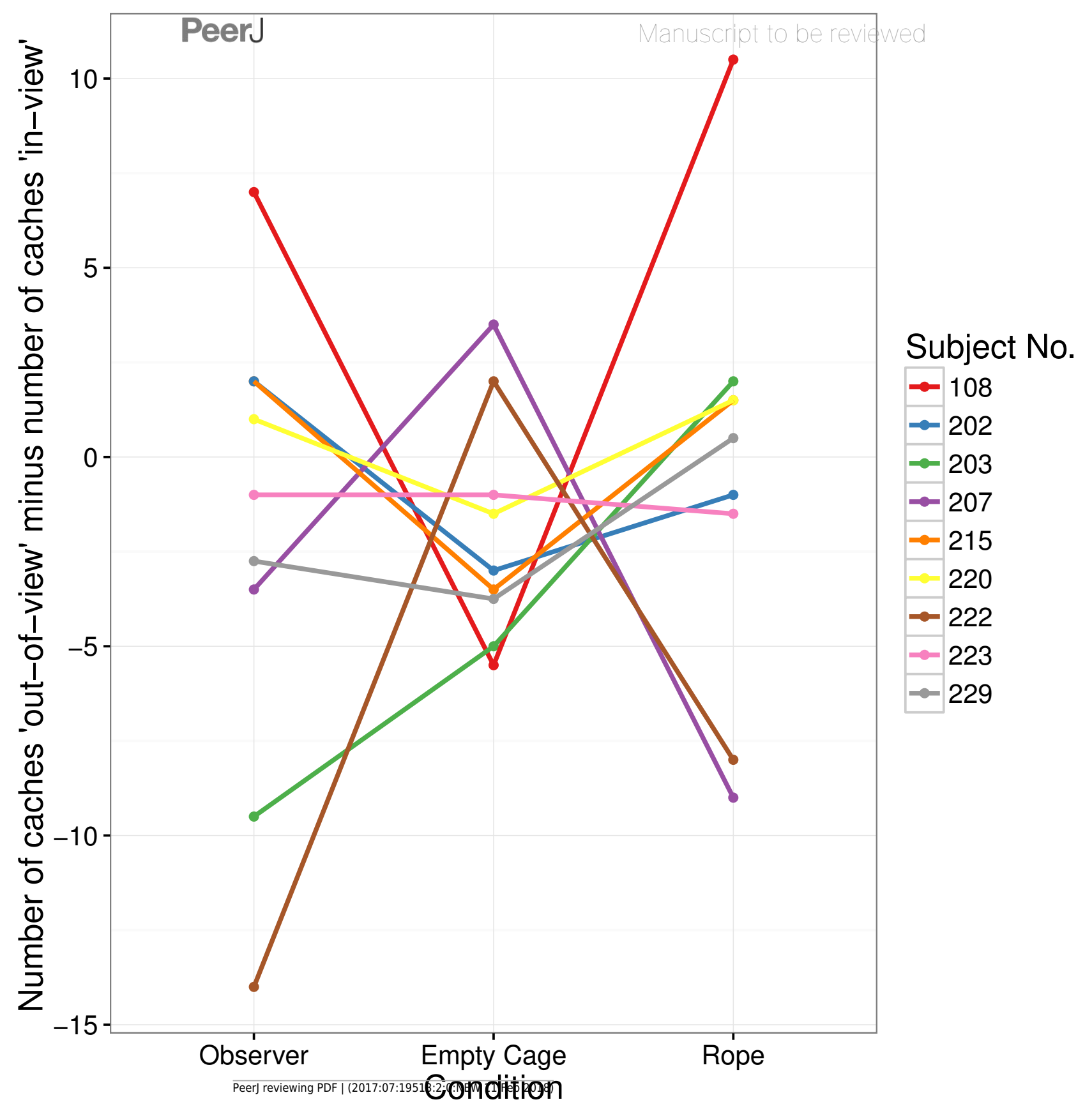


Figure 3 (on next page)

Proportion of re-cached items in Experiment 2.

Each data point indicates the proportion for one bird in each of the three conditions 


\section{Table $\mathbf{1}$ (on next page)}

Median number of items cached in Experiment 1. 


\begin{tabular}{lccc}
\hline & \multicolumn{3}{c}{ Median items cached (Min-Max) } \\
\cline { 2 - 4 } & Total & Out-of-view & In-view \\
\hline Obondition & & & \\
Ebserver & $6(1-30)$ & $4(0-8)$ & $2.5(0-22)$ \\
Rope & $13(1-39.5)$ & $5(0-17)$ & $8(0-22.5)$ \\
Trial No. & $9(1.5-21.5)$ & $5(0-16)$ & $6(1-10)$ \\
1 & & & \\
2 & $5.5(1.5-39.5)$ & $1(0-17)$ & $4.5(1-5-22.5)$ \\
3 & $8(1-24.5)$ & $4(0-16)$ & $3.5(0-17)$ \\
\hline
\end{tabular}

1 
Table 2 (on next page)

Median number of items cached and re-cached in Experiment 2. 


\begin{tabular}{lcc}
\hline & $\begin{array}{c}\text { Median items } \\
\text { cached (Min-Max) }\end{array}$ & $\begin{array}{c}\text { Median proportion of re- } \\
\text { cached items (Min-Max) }\end{array}$ \\
\hline Condition & $12.25(0-24)$ & $0.44(0.31-0.62)$ \\
Observer & $6.25(1-23)$ & $0.37(0.22-1)$ \\
Empty Cage & $6(0-38)$ & $0.42(0.29-1)$ \\
Rope & & \\
Trial No. & $12.25(1.5-38)$ & $0.45(0.19-1)$ \\
1 & $11.75(1-5-23)$ & $0.42(0.22-1)$ \\
2 & $8(2.5-24.0)$ & $0.41(0.28-0.6)$ \\
3
\end{tabular}

